\title{
Prevalence, Incidence and Ecological Determinants of Diabetic Retinopathy in Iran: Systematic Review and Meta-analysis
}

\author{
Golnoush Sadat Mahmoudi Nezhad ${ }^{1,2,3}$, MD; Reza Razeghinejad ${ }^{4}$, MD; Mohsen Janghorbani ${ }^{5,6}$, PhD; Alireza \\ Mohamadian $^{2,7}$, MD; Mohammad Hassan Jalalpour ${ }^{2}$, MD; Somaye Bazdar ${ }^{1,2}$, MD; Alireza Salehi ${ }^{1}$, MD; Hossein \\ Molavi Vardanjani ${ }^{1}, \mathrm{PhD}$
}

${ }^{1} \mathrm{MPH}$ Department, Shiraz University of Medical Sciences, Shiraz, Iran

${ }^{2}$ Poostchi Ophthalmology Research Center, Department of Ophthalmology, Shiraz University of Medical Sciences, Shiraz, Iran

${ }^{3}$ Glaucoma Division, Stein Eye Institute, David Geffen School of Medicine, University of California at Los Angeles, Los Angeles, CA, USA

${ }^{4}$ Glaucoma Service, Wills Eye Hospital, Philadelphia, PA, USA

${ }^{5}$ Department of Epidemiology and Biostatistics, School of Public Health, Isfahan University of Medical Sciences, Isfahan, Iran

${ }^{6}$ Isfahan Endocrine and Metabolism Research Center, Isfahan University of Medical Sciences, Isfahan, Iran

${ }^{7}$ Student Research Committee, Shiraz University of Medical Sciences, Shiraz, Iran

ORCID:

Golnoush Sadat Mahmoudi Nezhad: https://orcid.org/0000-0002-9233-9882

Hossein Molavi Vardanjani: https://orcid.org/0000-0001-7024-8894

\section{Abstract}

Purpose: To estimate the pooled prevalence and incidence of diabetic retinopathy (DR) in Iran and to investigate their correlations with the Human Development Index (HDI), healthcare access (i.e., density of specialists and sub-specialists), and methodological issues.

Methods: Electronic databases such as PubMed, Embase, Scopus, Web of Science, Google Scholar, and local databases were searched for cohort and cross-sectional studies published prior to January 2018. Prevalence and incidence rates of DR were extracted from January 2000 to December 2017 and random effects models were used to estimate pooled effect sizes. The Joanna Briggs Institute critical appraisal tool was applied for quality assessment of eligible studies.

Results: A total of 55,445 participants across 33 studies were included. The pooled prevalence $(95 \% \mathrm{Cl})$ of DR in diabetic clinics (22 studies), eye clinics (4 studies), and general population (7 studies) was $31.8 \%$ (24.5 to 39.2 ), $57.8 \%$ (50.2 to 65.3 ), and $29.6 \%$ (22.6 to 36.5 ), respectively. It was $7.4 \%$ (3.9 to 10.8 ) for proliferative DR and 7.1\% (4.9 to 9.4) for clinically significant macular edema. The heterogeneity of individual estimates of prevalence was highly significant. HDI $(P<0.001)$, density of specialists $(P=0.004)$, subspecialists $(P<$ 0.001), and sampling site $(P=0.041)$ were associated with heterogeneity after the adjustment for type of DR, duration of diabetes, study year, and proportion of diabetics with controlled HbA1C.

Conclusion: Human development and healthcare access were correlated with the prevalence of DR. Data were scarce on the prevalence of DR in less developed provinces. Participant recruitment in eye clinics might overestimate the prevalence of DR.

Keywords: Access to Health Care; Diabetic Retinopathy; Epidemiology; Human Development; Iran

J Ophthalmic Vis Res 2019; 14 (3): 321-335

\section{Correspondence to:}

Hossein Molavi Vardanjani, PhD. Department of MPH, Medical School, Shiraz University of Medical Sciences, Zand St., Shiraz 71348, Iran.

E-mail: H.molavivardanjani@gmail.com

Received: 22-12-2018

Accepted: 06-04-2019

\section{Access this article online}

Website:

https://knepublishing.com/index.php/JOVR

DOI:

10.18502/jovr.v14i3.4790 
This is an open access journal, and articles are distributed under the terms of the Creative Commons Attribution-NonCommercial-ShareAlike 4.0 License, which allows others to remix, tweak, and build upon the work non-commercially, as long as appropriate credit is given and the new creations are licensed under the identical terms.

How to cite this article: Mahmoudi Nezhad GS, Razeghinejad R, Janghorbani M, Mohamadian A, Jalalpour MH, Bazdar S, et al. Prevalence, incidence and ecological determinants of diabetic retinopathy in Iran: Systematic review and meta-analysis. J Ophthalmic Vis Res 2019;14:321-335.

\section{INTRODUCTION}

Diabetic retinopathy (DR) is the leading cause of vision loss in adults aged $20-74$ years, and remains one of the foremost causes of blindness and visual impairment worldwide. ${ }^{[1-4]}$ Despite significant development in the prevention and control of DR, the proportion of DR increased by $7.7 \%$ among all declining causes of blindness between 1990 and 2015. ${ }^{[5]}$ The prevalence of DR strongly correlates with both the duration of diabetes and the level of glycemic control. ${ }^{[6]}$ Therefore, timely management of DR stemming from screening programs, appropriate referral for treatment, and improving healthcare accessibility are important in preserving vision in diabetics. ${ }^{[7]}$

Although the treatment of DR can decrease the risk of visual loss by $60 \%$, it imposes a heavy cost to the healthcare system. ${ }^{[8]}$ Despite some improvements in diagnostic assessment and treatment options, ${ }^{[6]}$ the lack of qualified healthcare services along with a Westernized lifestyle have caused the burden of DR to be high and on the rise in developing countries. ${ }^{[7,8]} \mathrm{DR}$ is a pressing public health matter, probably due to suboptimal access to diabetes care services such as eye care professionals and eye care services, especially in low- to middle-income countries. ${ }^{[8]}$ Low human development might be another correlate of the increasing burden of DR. To the best of our knowledge, no study has investigated the correlation between human development and DR. The only study on this subject assessed the association between the Human Development Index (HDI) and the number of studies published on DR. ${ }^{[9]} \mathrm{HDI}$ is abstracted from income, education, and life expectancy markers and ranks areas into different levels of human development. ${ }^{[10]}$

Despite the high prevalence of diabetes, there are few reliable national studies on the incidence, prevalence, and correlates of DR in developing countries. $^{[11]}$ Of note, Iran is a country in transition, having a high variety of healthcare access options and human development as well as a huge variation in the prevalence and incidence of DR across its geographic regions. ${ }^{[12-14]}$ Therefore, besides assessing the prevalence and incidence of DR, their adjusted correlations with $\mathrm{HDI}$ and healthcare access were investigated in the current observational study.

\section{METHODS}

\section{Protocol and Registration}

The Meta-analysis of Observational Studies in Epidemiology (MOOSE) guidelines were followed. ${ }^{[15]}$ The study protocol was approved by the Shiraz University of Medical Sciences (ethical approval code: IR.SUMS.MED.REC.1397.256).

\section{Eligibility Criteria}

Observational studies (prospective or retrospective cohort and cross-sectional) were included if they provided sufficient information about the incidence and prevalence of DR and clinically significant macular edema (CSME). No restriction was applied on the year of publication or type of diabetes, and all studies published in Persian or English language were included. These two languages covered all studies published about the Iranian population and we did not find studies in other languages.

\section{Search Strategy}

We performed a systematic search for the prevalence and incidence of DR, summarized in Figure 1. PubMed, Scopus, Web of Science, Google Scholar, Embase, and the local databases of SID and Iran Doc were searched for articles published between January 2000 and December 2017. Our search was limited to studies related to Iran. The search terms included: "diabetes" or "diabetic" combined with "complication" or "retina" or "vision" or "visual" or "retinopathy" or "blindness" or "clinically significant macular edema" and "Iran" and "Epidemiology" or "incidence" or "prevalence" or "proportion". Review articles and their references were checked for additional studies. The gray literature evaluation was performed using international and regional 
congresses that were held during the study period around the world and specifically in Iran, and we selected and hand searched the abstract books that were obtainable as much as possible. We also searched university websites for thesis and reports that were related to the subject during the study period. References of all included studies were also searched for potentially eligible studies. In cases where the full text of an article was unavailable, the corresponding author was contacted. Documents were catalogued using Endnote X4.

\section{Study Selection}

In case of repeated publications from one study, the newest publication was included. Titles, abstracts, and the full texts of retrieved articles were reviewed independently by two experts in the field and eligible articles were selected. Studies on children, pregnant women, or nonIranian populations were excluded as well as experimental studies, secondary studies, letters, case-reports, case-series, commentaries, and editorials. Clinical trials were also excluded. Studies with a non-representative sample or irrelevant comorbidities were also excluded. Also, in the case of disagreement regarding exclusion or inclusion of a study, a consensus was reached through discussion between the authors.

\section{Data Extraction and Risk of Bias Assessment}

We performed a comprehensive literature review and designed a conceptual framework. An excel (MS Office) data sheet based on clinical principles was prepared for data extraction using our framework. The following variables were included in the data extraction form: first author, publication year, study year, study location (i.e., province/district), urbanization ratio (number of participants from the urban area/number of participants from the rural area; based on the data presented in each individual study), sampling site (diabetes clinic, eye clinic, or general population), sampling design (random, multistage, convenient, unknown), study design (cross-sectional, cohort), sample size (overall and for subgroups), age range (or mean age), duration of study, gender (or female/male ratio), diagnostic methods of DR, ${ }^{[16]}$ proportion of diabetics (in population-based studies), type of diabetes (or type
I/type II ratio), type of DR (i.e., non-proliferative diabetic retinopathy [NPDR]) and its stage (mild, moderate, or severe), proliferative diabetic retinopathy (PDR), CSME, mean HbA1C (overall, among DR patients, and among non-DR patients), number of DR patients (or estimated prevalence and its standard error), number of incident cases of DR (or cumulative incidence rate; only for cohort studies), number of person-years of follow-up (in cohort studies), duration of DM (overall, among DR patients, and among non-DR patients), mean age at the onset of DM, mean duration of DR, and proportion of patients with newly diagnosed DM. The aforementioned data were extracted (if available) and $25 \%$ of the extracted data were randomly cross-checked by another author. Also, in the case of disagreement regarding data extraction, a consensus was reached through discussion between the authors. Since several studies had not reported exact values for some variables, more than 30 disagreements on the most appropriate estimates for these missing values were discussed in the team.

The Joanna Briggs Institute (JBI)'s critical appraisal too ${ }^{[17]}$ was applied for quality assessment of eligible studies. Quality assessments and critical appraisals were performed by two different authors independently. In the case of disagreement regarding the quality score, a consensus was reached through discussion between the authors (4 studies out of the included 33 studies).

\section{Data on Human Development and Healthcare Access}

The density of specialists and subspecialists (numbers of specialists and subspecialists in the healthcare system of each province to the total population in that province) was retrieved from a recent reliable report by Haghdoost et al ${ }^{[18]}$ as indices of healthcare access. Density ratios were categorized into quantiles. Haghdoost et al gathered the number of physicians based on the questionnaires filled out by medical universities all around Iran. Their study was a part of a project to define the national treatment map of Iran in 2025 (Naghsh-e Rahe Darman-e Iran). In order to control the precision of completing the questionnaire, their data was crosschecked with the Medical Registry Information System and different medical insurance companies. ${ }^{[18]}$ 


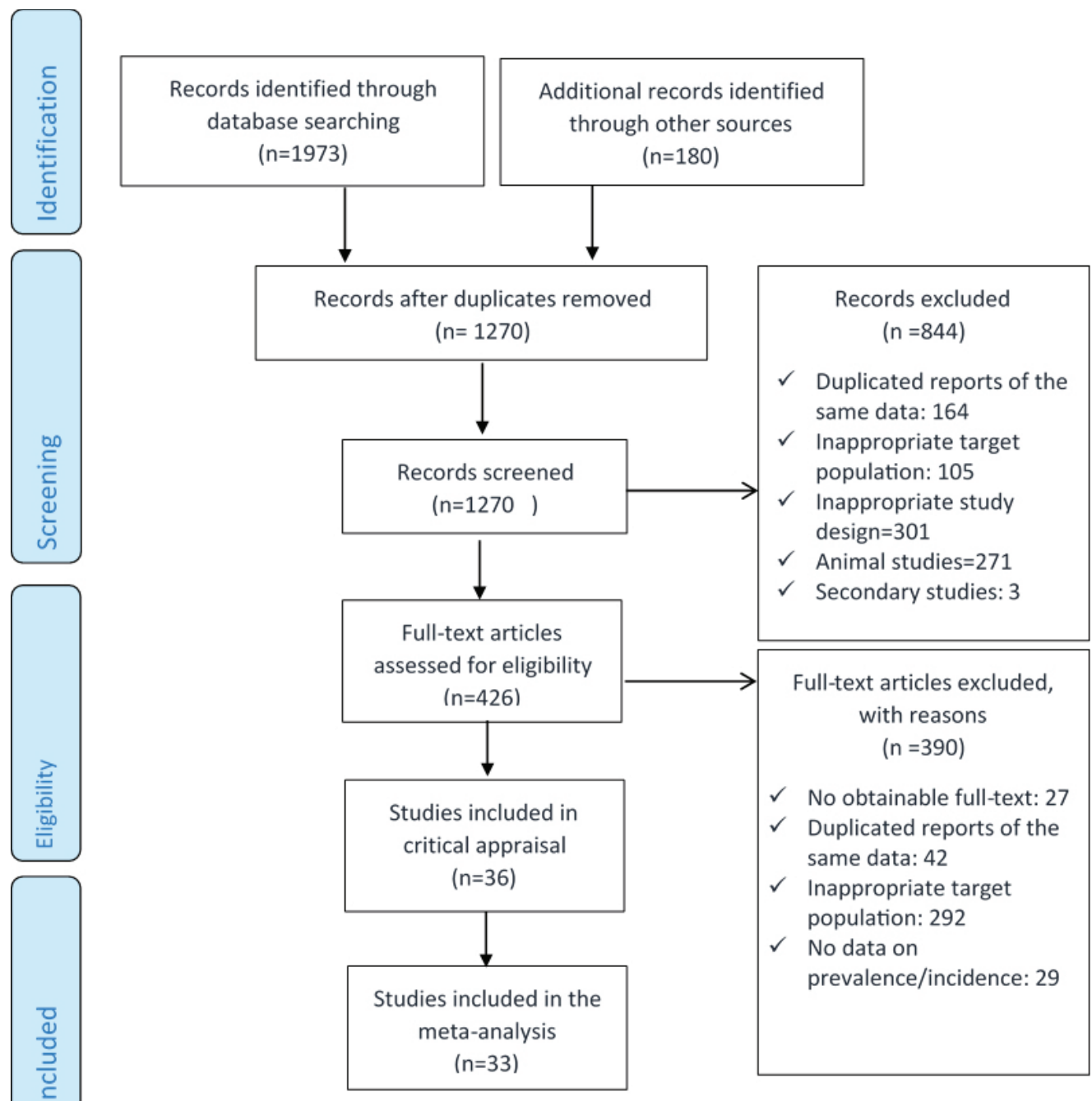

Figure 1. Flow diagram summarizing the systematic search and review process for identifying evidence regarding the prevalence and diabetic retinopathy. Inclusion and exclusion criteria are provided in the text.

HDI indices (less developed, moderately developed, and developed) were retrieved for each province according to the study performed by Safaeipour et $\mathrm{al}^{[12]}$ and used as an independent variable in meta-regression modeling.

\section{Data Preparation and Statistical Analysis}

Point estimates of prevalence were extracted or calculated as the number of patients with DR divided by the number of patients with DM. Additionally, prevalence estimates of DR in the population, regardless of diabetic status, were estimated as the number of participants with DR divided by the number of total participants in populationbased studies. A 95\% confidence interval (Cl) for all individual point estimates of prevalence was estimated where it was not mentioned. ${ }^{[19]}$ Cumulative incidence proportions (per 100 person-years) were calculated as the number of new DR cases divided by the number of at-risk person-years (i.e., number of study subjects multiplied by number of follow-up years).

To ensure the independence of point estimates in primary studies as well as to prevent repeated counting of participants in primary studies, only one of the overall or subgroup point estimates of each primary study was included in the meta-analysis and meta-regression models. The heterogeneity of individual estimates of prevalence was assessed according to the I-square statistic above $50 \% .{ }^{[20]}$ In cases of high heterogeneity, correlates were 
conceptualized. Then, the significance and magnitude of their correlation were investigated using the random-effects meta-regression technique.

Subgroup analyses were performed according to the most important correlates of heterogeneity if applicable (i.e., sampling site, geographical location, $\mathrm{HDI}$ and healthcare access). Due to persistent heterogeneity (even after subgroup analysis), individual estimates of prevalence and incidence were pooled using the DerSimonian and Laird randomeffects modeling method. Publication bias was investigated using Begg's and Egger's tests. Data analysis and calculations were performed using Stata software, version 11.2 (Stata Corporation, College Station, TX, USA). A two-sided $P<0.05$ was considered as statistically significant. We also provided appropriate tables and graphs for showing our results (i.e., included studies, shortage in studies, methods applied for the diagnosis of DR by primary studies, study flowchart, and study forest plot by HDI categories). The study protocol was registered in the International Prospective Register of Systematic Reviews (PROSPERO), under the registration number CRD42018104626.

\section{RESULTS}

The initial search resulted in 2,153 records. Of these, 426 records were included in the fulltext review process, and finally, 33 studies were included in the meta-analysis.

\section{Overview of Included Studies}

Thirty cross-sectional and three cohort studies were included in the analysis, representing an overall number of 55,445 diabetic patients including 17,155 patients with DR [Table 1]. Among cross-sectional studies, seven had a populationbased sampling design representing 24,623 participants including 5,657 diabetic patients and 2,049 patients with DR. Included cohort studies represented 1,174 diabetic patients (equivalent to 5,400 person-years) and 613 incident cases of DR.

\section{Assessment of Heterogeneity in Individual Estimates for Prevalence of Diabetic Retinopathy}

In cross-sectional studies conducted in diabetes clinics, individual estimates for the prevalence of DR were significantly heterogeneous for overall diabetics (I-square, 99.5; $P<0.001$; the heterogeneity was similar between genders [I-square, 99.4; $P<0.001]$ ). Also, individual estimates for the prevalence of DR were highly heterogeneous in studies conducted in eye clinics (I-square, 98.0; $P<0.001$ ) and population-based studies (I-square, 95.7; $P<0.001)$. Additionally, in three cohort studies, the estimates of individual cumulative incidence of DR were statistically heterogeneous with a highly significant I-square of $97.9(P<0.001)$.

\section{Determinants of DR Prevalence (Correlates of Heterogeneity of Individual Estimates)}

According to the random-effects meta-regression model, HDI (as an ordinal variable of tertile of HDI; adjusted OR: 0.12, 95\% Cl: 0.05 to 0.34 , $P<0.001$ ), density of specialists (as an ordinal variable including quintile of density ratio; adjusted OR: $1.13,95 \% \mathrm{Cl}: 1.04$ to $1.23, P=0.004)$, density of subspecialists (as an ordinal variable including quintile of density ratio; adjusted OR: $0.85,95 \%$ $\mathrm{Cl}$ : 0.78 to $0.91, P<0.001$ ), type of DR (reference is PDR; adjusted OR: $1.30,95 \% \mathrm{Cl}: 1.10$ to $1.42, P$ $<0.001$ ), duration of diabetes (adjusted OR: 1.05, 95\% Cl: 1.04 to $1.07, P<0.001$ ), site of study sampling (reference is population-based sampling; adjusted OR: 1.09, 95\% Cl: 1.02 to $1.17, P=0.041$ ), study year (adjusted OR: 0.97, 95\% Cl: 0.96 to $0.98, P<0.001$ ), and proportion of diabetics with controlled HbA1C (adjusted OR: 0.92, 95\% Cl: 0.87 to $0.97, P=0.005$ ) were significantly associated with the heterogeneity of individual estimates of DR prevalence.

Risk of bias was not associated with heterogeneity $(P=0.683)$. Type of diabetes was not a significant determinant of heterogeneity for individual estimates of DR prevalence $(P=0.10)$.

Due to the lack of enough data to achieve individual estimates of cumulative incidence, metaregression modeling for cumulative incidence was not applicable. 


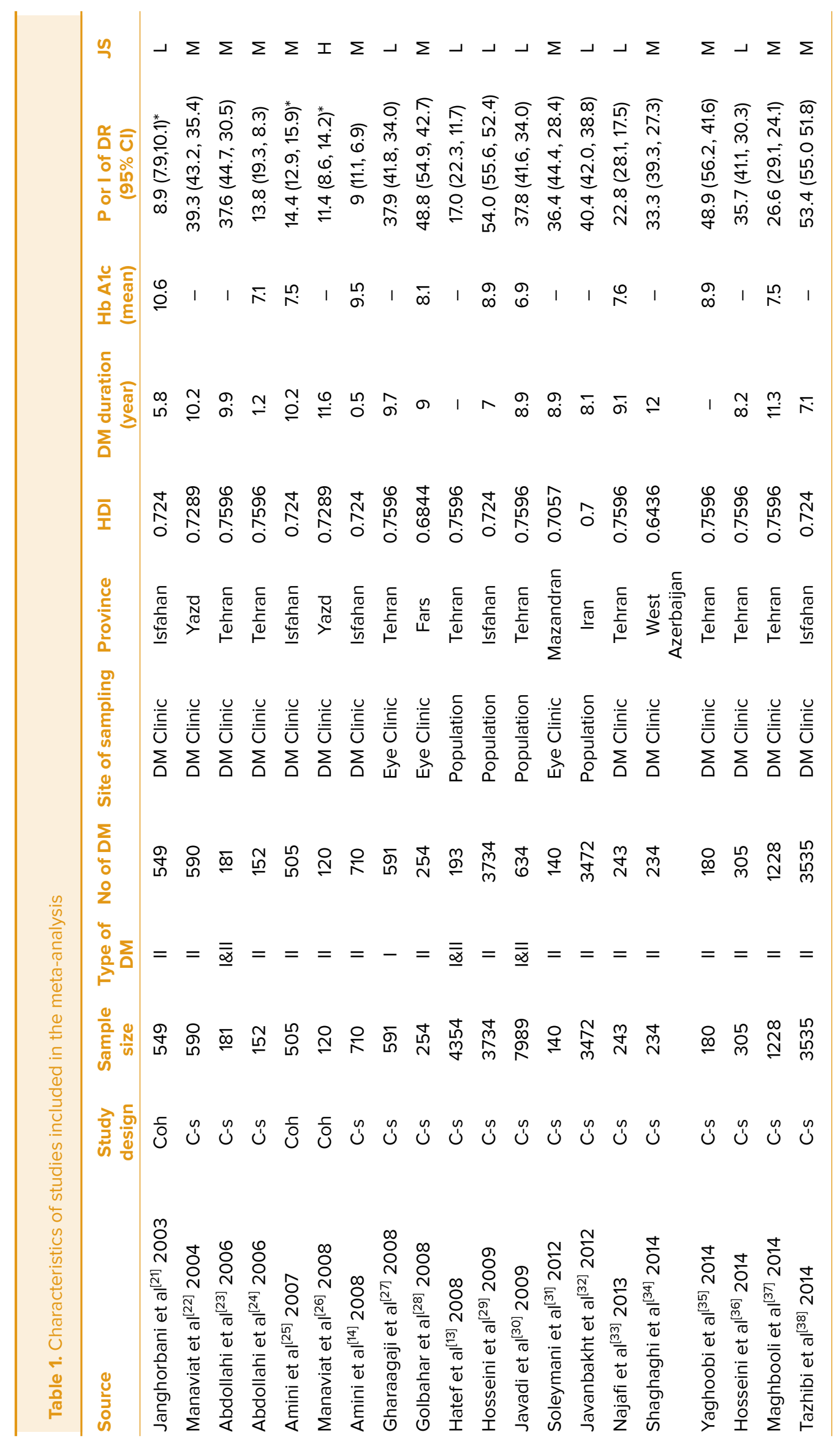




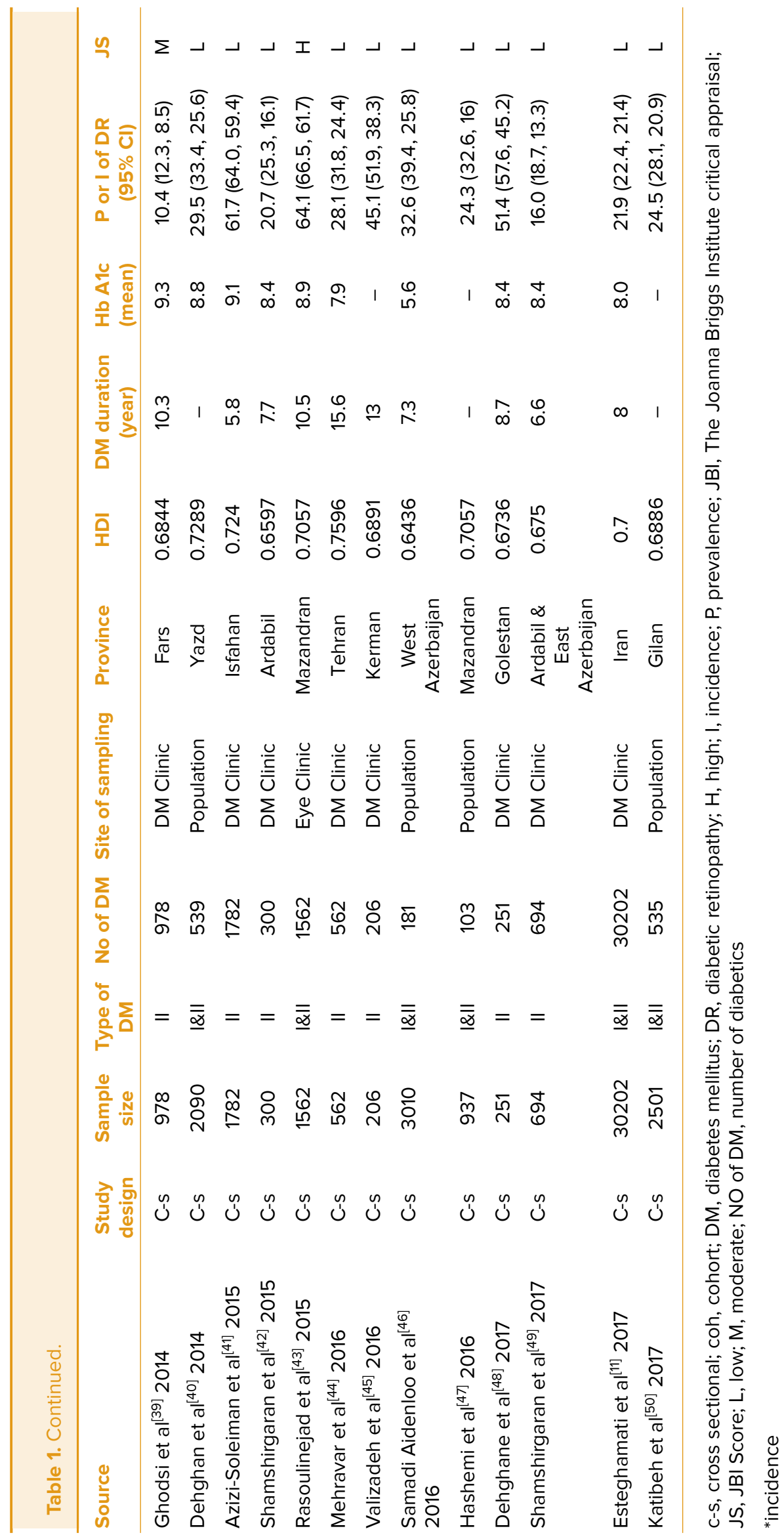




\section{Prevalence of DR among Diabetic Patients}

Due to high heterogeneity, pooled prevalence estimates might have some extent of bias from an epidemiological point of view. The range for overall prevalence of DR among diabetics was 9.0-63.4\%. (The pooled estimate of prevalence using randomeffect model was 33.6\% [95\% Cl: 27.9, 39.2], 40.6\% [95\% Cl: 28.9, 52.3], and 35.7\% [95\% Cl: 26.0, 39.4] overall in males and females, respectively [Figure 2]).

The pooled prevalence of NPDR, mild NPDR, moderate NPDR, and severe NPDR among diabetic patients were $24.8 \%$ (95\% Cl: 18.7, 30.9), 14.1\% (95\% Cl: 9.1, 19.2), 8.9\% (95\% Cl: 4.7, 13.0), and 3.3\% (95\% Cl: 2.0, 4.6), respectively. The pooled prevalence of PDR and CSME were $7.4 \%(95 \% \mathrm{Cl}: 3.9,10.8)$ and $7.1 \%$ (95\% Cl: 4.9, 9.4), respectively.

Due to significant heterogeneity among studies, the analysis was performed through subgroup analysis based on the most important correlates of heterogeneity.

\section{Prevalence of DR among Patients Referred to Diabetic Clinics}

The overall pooled prevalence rate of DR among diabetic patients was $31.8 \%$ (95\% Cl: $24.5,39.2)$ and $39.1 \%$ (95\% Cl: $23.5,54.6)$ among male subjects and $34.6 \%$ (95\% Cl: 23.2, 45.9) among female subjects.

The pooled prevalence rates of NPDR, mild NPDR, moderate NPDR, and severe NPDR among diabetic patients were $21.0 \%$ (95\% Cl: 12.7, 29.3), $11.1 \%$ (95\% Cl: $3.3,18.9), 5.6 \%$ (95\% Cl: $0.7,11.9)$, and $2.2 \%$ (95\% Cl: 1.2, 3.3), respectively. The pooled prevalence rates of PDR and CSME among diabetic patients were $2.9 \%(95 \% \mathrm{Cl}: 1.3,4.5)$ and $7.4 \%(95 \%$ Cl: 6.1, 8.6).

The pooled estimate of prevalence of DR based on studies including only type II diabetics was $33.3 \%$ (95\% Cl: 24.4, 42.2), whereas, it was $22.6 \%$ (95\% Cl: $12.8,32.3)$ in those with both types of diabetes.

The pooled prevalence rates of DR in the central, northeast, northwest, southeast, and southwest geographic regions of Iran were $42.9 \%(95 \% \mathrm{Cl}$ : 30.4, 55.3), 51.4\% (95\% Cl: 45.2, 57.5), 26.6\% (95\% Cl: $18.6,34.6), 45.1 \%$ (95\% Cl: 40.3, 49.9), and 10.4\%
(95\% Cl: 8.7, 12.5), respectively, and 30.8\% (95\% Cl: 26.6, 35.0) for Tehran (the capital city of Iran).

\section{Prevalence of DR among Patients Referred to Eye Clinics}

The overall pooled prevalence of DR among diabetic patients was $57.8 \%(95 \% \mathrm{Cl}: 50.2,65.3)$ and $63.0 \%$ (95\% Cl: $60.4,65.6)$ among males and 61.3\% (95\% Cl: 57.0, 65.7) among females. The pooled prevalence rates of NPDR and PDR were 29.0\% (95\% Cl: 23.4, 34.5) and 19.4\% (95 \% Cl: 14.6, 24.3), respectively. The pooled estimate of DR prevalence was $48.8 \%$ (95\% Cl: $42.7,55.0)$ in studies including only type II diabetics and $64.6 \%$ (95\% Cl: 62.2, 67.0) in studies including both types of diabetes. The pooled prevalence rates of DR in the north and southwest geographical regions of Iran were $58.4 \%(95 \% \mathrm{Cl}: 51.3,65.5)$ and $48.8 \%$ (95\% Cl: 44.5, 53.2), respectively, and 39.1\% (95\% Cl: 27.2, 51.0) in Tehran.

\section{Prevalence of DR among Patients in Population-based Studies}

The overall pooled prevalence of DR was $29.6 \%$ (95\% Cl: $22.6,36.5)$ and $36.8 \%(95 \% \mathrm{Cl}: 30.5$, 43.2) among male subjects and $29.0 \%$ (95\% Cl: 24.6, 33.6) among female subjects. The pooled prevalence rates of DR in the central, north, and northwest geographical regions of Iran were 29.9\% (95\% Cl: 26.4, 33.4), 24.4\% (95\% Cl: 21.3, 27.5), and $32.5 \%$ (95\% Cl: 27.7, 37.4), respectively, and 32.8\% (95\% Cl: 22.3, 43.3) in Tehran.

\section{Prevalence of DR in the General Population}

The overall pooled prevalence of DR (according to the population-based studies) was $3.6 \%(95 \% \mathrm{Cl}$ : $2.4,5.0)$ and $3.5 \%(95 \% \mathrm{Cl}: 1.5,6.4)$ among male subjects and $3.6 \%(95 \% \mathrm{Cl}: 2.1,5.6)$ among female subjects.

\section{Cumulative Incidence Rate of DR in Diabetics}

The overall pooled cumulative incidence rate (per 100 person-years) of DR was $11.7 \%$ (95\% Cl: 8.0 , $15.9)$ and $9.6 \%(95 \% \mathrm{Cl}: 7.8,11.8)$ among male subjects and $8.7 \%(95 \% \mathrm{Cl}: 7.5,10.0)$ among female 


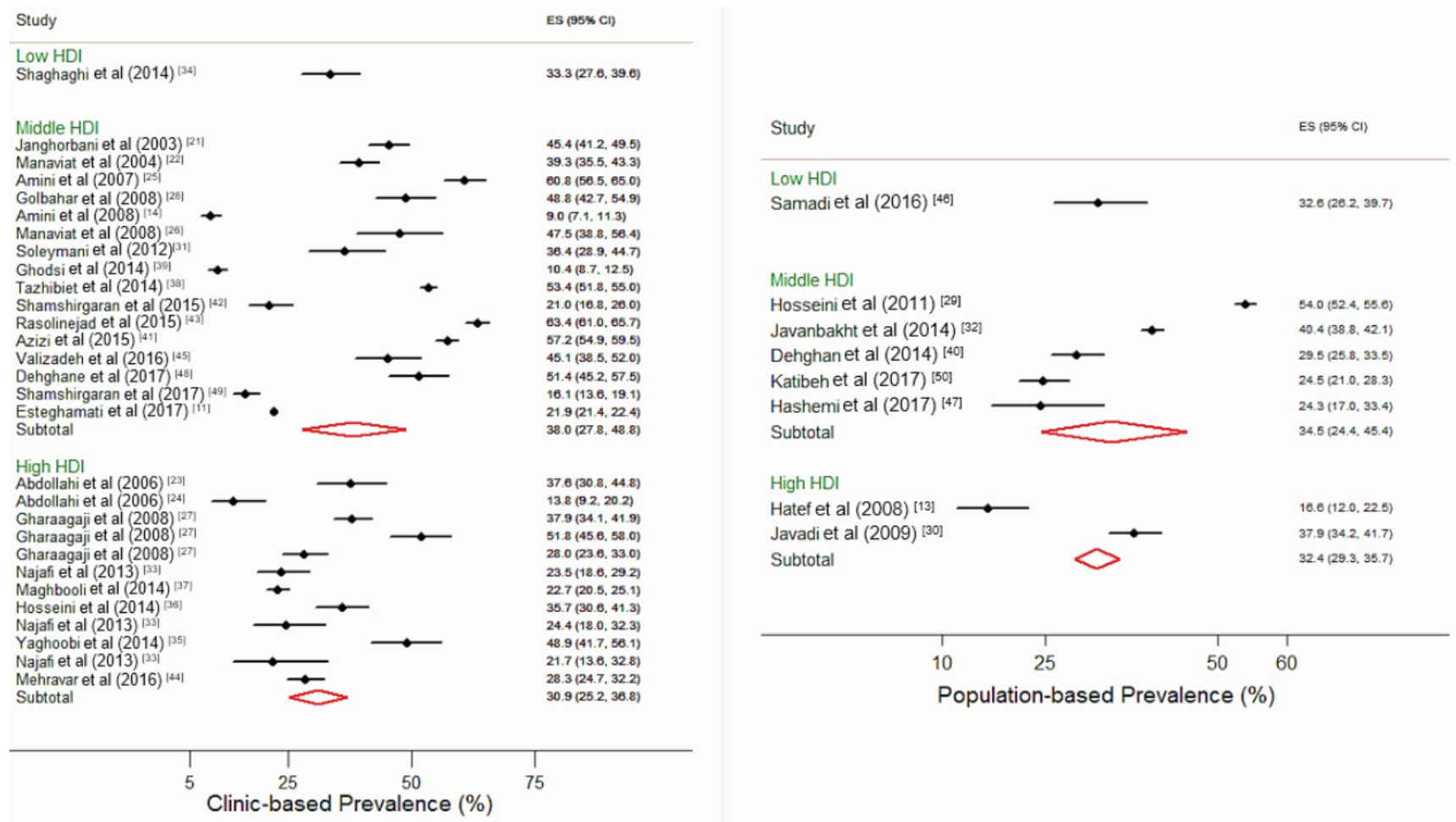

Figure 2. Forest plot of diabetic retinopathy prevalence by Human Development Index categories and source of data (clinic vs population-based studies). Cl, confidence interval; ES, estimation of diabetic retinopathy prevalence (\%); HDI, Human Development Index.

subjects [Figure 3]. The pooled cumulative incidence rates (per 100 person-years) for NPDR and PDR were $11.7 \%(95 \% \mathrm{Cl}: 9.1,14.9)$ and $0.2 \%(95 \%$ $\mathrm{Cl}: 0.0,1.1)$, respectively.

\section{More Details on the Association of HDI and the Prevalence of DR}

The estimation of linear correlation coefficient between $\mathrm{HDI}$ and the prevalence of DR among diabetics was -0.18 with a $P$ of 0.029 (a relatively low but significant linear correlation) [Figure 4].

\section{Reporting and Methodological Shortcomings}

One study used the survey data analysis technique to consider multistage sampling design. ${ }^{[50]}$ Two studies reported age-adjusted estimates. ${ }^{[30,40]}$ Only 33.3\% ( $n=11)$ of the studies reported their DR results by gender [Table 2].

\section{Assessment of DR}

According to the current results, $54.54 \%(n=18)$ of studies performed indirect ophthalmoscopy with pupillary dilatation for the evaluation of DR. Others probably used the same method; however, this matter was not clearly stated [Table 3].

\section{Publication Bias}

There was no significant publication bias (Begg's test $P=0.824$; Egger's test $P=0.075$ ) in the current study.

\section{DISCUSSION}

In the current study, the pooled prevalence and incidence of DR including PDR and NPDR among Iranian diabetic subjects referred to diabetes clinics and eye clinics and the pooled prevalence of DR among diabetics retrieved form populationbased studies were determined based on the English language studies only. Persian articles were excluded due to poor quality score in our 


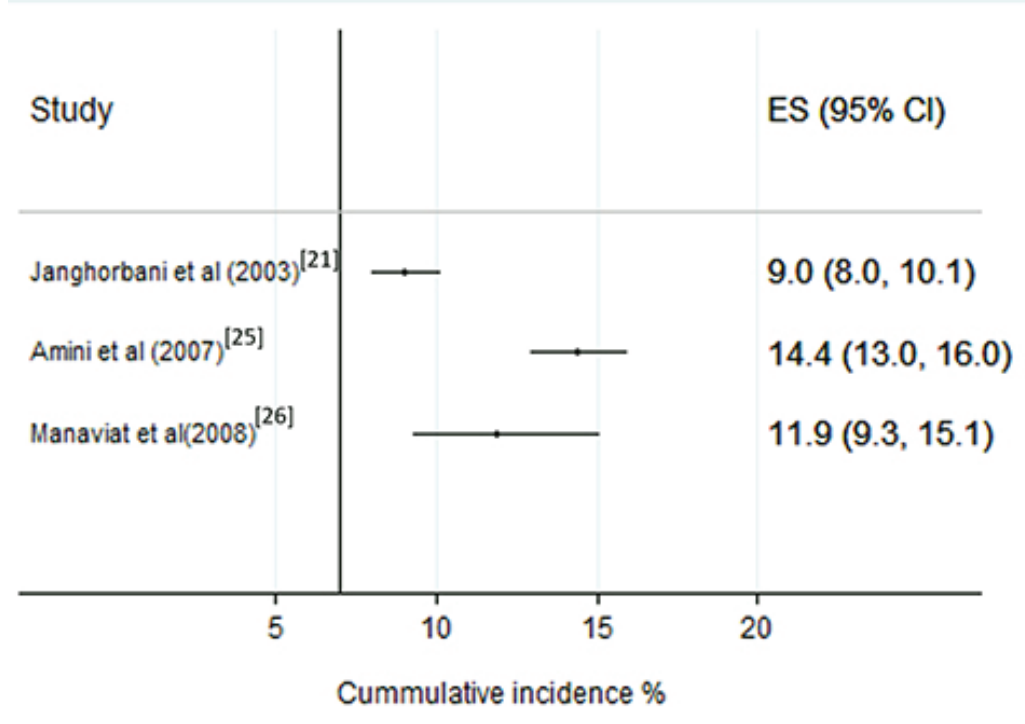

Figure 3. Forest plot of cumulative incidence rate of diabetic retinopathy. $\mathrm{Cl}$, confidence interval; ES, estimation of diabetic retinopathy incidence (\%)

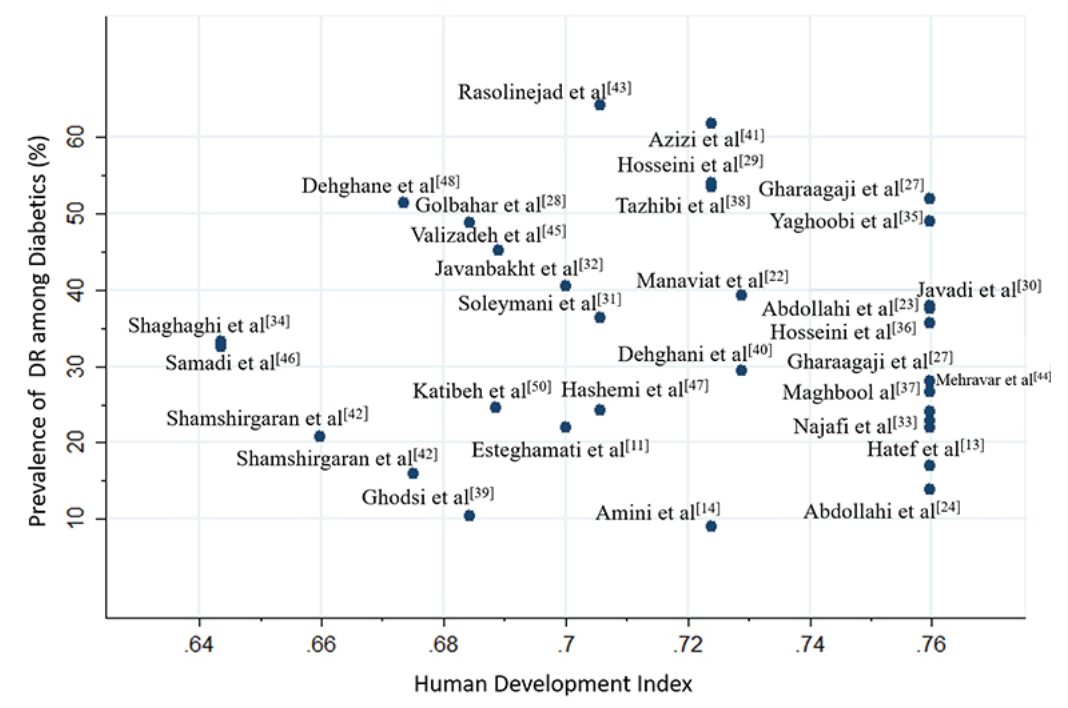

Figure 4. Scatter plot of the linear correlation of prevalence and the Human Development Index. DR, diabetic retinopathy

assessment. We found no publication bias among the included studies. The human development, access to subspecialists and specialists, and site of study sampling had a correlation with the prevalence of DR among diabetics.

Studies from other parts of the world reflect significant differences in the prevalence of DR depending on the factors such as ethnicity, demography, and access to healthcare systems. ${ }^{[51,52]}$ According to the current results, an increase in HDI was independently correlated with lower DR prevalence. In order to avoid the bias of ecological inference fallacy, this finding could only be interpreted from an ecological point of view. Therefore, we cannot directly relate the DR prevalence in individuals to the HDI in each province. However, HDI may potentially be a good index of quality of diabetes care in developing countries such as Iran at a national level. This finding is consistent with previous evidence from low-income regions of developed countries. ${ }^{[53]}$ The prevalence of DR and PDR/NPDR ratio were higher in eye clinics in 
Table 2. Number (\%) of studies reporting important criteria required in epidemiological studies on diabetic retinopathy

\begin{tabular}{lcc}
\hline Reportable variables & Studies on subjects with DM & $\begin{array}{c}\text { Studies on subjects with DR } \\
\text { N (\%) }\end{array}$ \\
\hline Study year & $26(78.7)$ & $26(78.7)$ \\
Source of recruitment & $33(100)$ & $33(100)$ \\
Sample size & $33(100)$ & $30(90)$ \\
Gender & $33(100)$ & $11(33.3)$ \\
Age (Mean \pm SD) & $22(66.6)$ & $16(48.4)$ \\
Dividing sample of study by age groups & $1(3.0)$ & $1(3.0)$ \\
Type of DM & $27(81.8)$ & $27(81.8)$ \\
Proportion of DM types ( $n=11)^{*}$ & $2(18.2)$ & $11(33.3)$ \\
Duration of DM & $14(42.4)$ & 0 \\
Proportion of patients with controlled DM & $1(3.0)$ & $14(42.4)$ \\
Type of retinopathy & $14(42.4)$ & $3.0(9.0)$ \\
Mean age at diagnosis of Diabetes & $3.0(9.0)$ & $15(45)$ \\
HbA1c (Mean \pm SD) & $20(60.6)$ & $2(6)$ \\
Age-Gender adjusted prevalence of retinopathy & $2(6)$ & $1 \%$ \\
\hline
\end{tabular}

*Only 11 studies included both types of DM. The others reported one type of the DM in their sample size. Proportion of the DM types in these 11 studies is considered to be an important factor for both diabetics and patients with DR.

DM, diabetes mellitus; DR, diabetic retinopathy; HbA1c, hemoglobin A1c; N, number; SD, standard deviation

Table 3. Findings about diagnostic methods for diabetic retinopathy

\begin{tabular}{lcc}
\hline Diagnostic method & Number of studies & Percent \\
\hline Indirect ophthalmoscopy & 11 & 33.33 \\
Indirect ophthalmoscopy and fluorescein angiography & 3 & 9.09 \\
Indirect ophthalmoscopy and fundus photography & 4 & 12.12 \\
Questionnaires/Records & 7 & 21.21 \\
Fundoscopic and angiographic findings & 1 & 3.03 \\
Slit-Lamp biomicroscopy of the posterior pole using contact lens & 1 & 3.03 \\
Eye examination and fundus photography & 1 & 3.03 \\
Ophthalmic examination (not mentioned exactly) & 5 & 15.15 \\
Total number of studies & 33 & 100
\end{tabular}

comparison to the DM clinics. It might be due to the higher detection rate of DR, especially PDR in eye clinics. ${ }^{[54-56]}$ In other words, more advanced patients are usually referred to the eye clinics.

The density of subspecialists was negatively correlated with the prevalence of DR. This could be due to health literacy, greater access to healthcare services in regions where subspecialists work, leading to more effective diabetes control. This finding is in accordance with previous reports. ${ }^{[51,52,57]}$ Of note, many confounding factors such as social factors could be associated with the density of subspecialists and prevalence of DR. It might also be due to the interest of subspecialists to be in areas with higher socioeconomic status in which inhabitants may also have a better access to 
healthy food, causing more controlled DM and less prevalence of DR.

The pooled population-based prevalence of DR among diabetics was close to the maximum estimates of DR prevalence reported in developing countries such as Pakistan (29\% vs $25-29 \%$ ), while it was lower than the prevalence reported in developed countries. ${ }^{[58-62]}$ Population aging and Westernized lifestyle could have been among the factors increasing the prevalence of diabetes and DR in Iran and other developing countries. ${ }^{[11,58,63]}$ In addition, the higher prevalence of DR among male subjects could be attributed to lifestyle habits such as cigarette smoking, which is consistent with studies conducted in India and Nepal. ${ }^{[58,59]}$

The pooled prevalence of DR among diabetics referred to eye clinics was significantly higher than that in diabetes clinics. Routine screening by an ophthalmologist is not a common practice in developing countries. ${ }^{[64]}$ Thus, this higher prevalence may be due to higher rates of referral for patients presenting with visual symptoms. There is a need for improving screening programs in primary healthcare services and communication between primary healthcare providers and ophthalmologists to ensure diabetics receive timely ophthalmic examinations.

Based on the results of the current study, the estimation of DR prevalence among diabetics in eye clinics may not be a good indicator of DR prevalence among diabetics; however, a comparison of DR prevalence in diabetic and eye clinics could help determine the sensitivity and specificity of referrals to eye clinics by clinicians and over/underutilization of eye care in developing countries.

Although, overall and gender-specific pooled prevalence rates of $D R$ among diabetics referred to diabetes clinics were relatively higher than those in population-based studies, these differences were not meaningful. In addition, the female/male ratio of estimated DR prevalence in DM clinics studies was almost equal to the population-based studies (88\% vs $80 \%$ ). Therefore, considering the difficulties in conducting robust population-based studies, it is reasonable to estimate the prevalence of $D R$ in diabetes clinics, especially in developing counties such as Iran.

The pooled estimate of DR incidence among diabetics in Iran was less than recent reports from developed countries such as Canada, the
UK, and Spain. ${ }^{[61,65,66]}$ Moreover, the estimated incidence was relatively higher than India, South Korea, and Denmark. ${ }^{[67-69]}$ According to the current study, the available data on the incidence of DR in Iran is inadequate; further studies are needed to determine the incidence of DR in Iran.

Among the eligible studies included in the current meta-analysis, only one study reported the prevalence of DR among Type I diabetics. ${ }^{[27]}$ Accordingly, the pooled estimates provided in the current study are mostly representative of the prevalence of DR among Type II diabetics. More studies on the incidence and prevalence of DR among Type I diabetics are needed.

The results of this study showed that there is a lack of research on the prevalence of DR in less developed provinces, such as Sistan and Balochistan, Bushehr, Hormozgan, Khorasan, Khozestan, Kermanshah, Kordistan, Kohkiloieye, Chaharmahal and Bakhtiyari, and Lorestan. Missing data from these provinces could have affected the results of the current study. In addition to the paucity of data in some provinces, there is a significant heterogeneity among studies, which means that making an accurate single prevalence estimate of DR is not possible. The pooled prevalence of DR was $29 \%$. However, the prevalence rate varied from $9 \%$ to $64.1 \%$. Thus, it might not be completely representative of the actual DR prevalence.

In addition to the aforementioned factors, the method used for detecting DR is a major factor that can influence prevalence estimates. It is crucial to know which specific diagnostic method (i.e., dilated fundus examination, direct or indirect ophthalmoscopy, digital imaging, etc.) was used in each study. ${ }^{[70]}$ The ophthalmic examination methods were not adequately explained in at least 12 included studies. In the course of DR, patients might develop maculopathy with no significant change in vision and may not seek medical care. Optical coherence tomography (OCT) is an important test in the evaluation and management of diabetic macular edema and can detect subclinical macular edema. Missing OCT evaluation could lead to many diabetics remaining undiagnosed in the early stage of DR. ${ }^{[71]}$

The current study has some limitations. The pooling of data from different sources introduced potential sources of heterogeneity that could impact accuracy. Various studies could have different inclusion criteria, sample selection, or study 
protocols. For example, the sample from a diabetes clinic differs from an eye clinic or population-based studies. The pooled estimate of DR prevalence by gender in the current study was sometimes not compatible with the total prevalence in the subgroups, which was due to the lack of reporting DR by gender in some studies. Studies in which the diagnosis of DM was based on patient selfreport, without lab test confirmation, could have led to an overestimation of DR prevalence due to the exclusion of undiagnosed diabetes from the study sample. Although it is desirable to have the HDI and density of specialists and subspecialists at the time of each study to calculate the correlations and perform the analyses, only two up-to-date and available studies were used for estimating the value of both mentioned variables. Also, the absence of studies from the eastern, western, and southern regions of Iran could have also affected the validity and generalizability of the current findings. Although our findings on the prevalence and incidence of DR may not be generalizable to all countries, it could be useful for developing countries, especially regions with similar socioeconomic, demographic, cultural, and geographic conditions.

\section{SUMMARY}

Despite the scarcity of research in less developed regions, a reasonable estimate for the prevalence of DR among Iranian diabetic subjects is around $30 \%$ (29\% among female and 37\% among male subjects). HDI, density of specialists and subspecialists, and sampling site were independently correlated with the prevalence of DR in Iran. The most reliable evidence on DR prevalence is likely to be retrieved from diabetes clinics in developing countries. Furthermore, providing a list of essential items for reporting the descriptive epidemiology of DR and performing studies in less developed regions could generate stronger evidence for health policy programs.

\section{Acknowledgments}

This study was supported by the Shiraz University of Medical Sciences and approved under code 97-01-01-16865. The study was extracted from the thesis written by Golnoush Sadat Mahmoudi Nezhad for MD-MPH degree.

\section{Financial Support and Sponsorship}

Nil.

\section{Conflicts of Interest}

There is no conflict of interest.

\section{REFERENCES}

1. Buch $H$, Vinding $T$, La Cour M, Appleyard M, Jensen GB, Nielsen NV. Prevalence and causes of visual impairment and blindness among 9980 Scandinavian adults: the Copenhagen City Eye Study. Ophthalmology 2004;111:5361.

2. Brazionis L, Jenkins A, Keech A, Ryan C, Brown A, Boffa $J$, et al. Diabetic retinopathy in a remote Indigenous primary healthcare population: a Central Australian diabetic retinopathy screening study in the Telehealth Eye and Associated Medical Services Network project. Diabet Med 2018;35:630-639.

3. Tan GS, Gan A, Sabanayagam C, Tham YC, Neelam K, Mitchell $P$, et al. Ethnic differences in the prevalence and risk factors of diabetic retinopathy: the Singapore epidemiology of eye diseases study. Ophthalmology 2018;125:529-536.

4. Cheung N, Mitchell P, Wong TY. Diabetic retinopathy. Lancet 2010. 10;376:124-136.

5. Flaxman SR, Bourne RR, Resnikoff S, Ackland P, Braithwaite T, Cicinelli MV, et al. Global causes of blindness and distance vision impairment 1990-2020: a systematic review and meta-analysis. The Lancet Glob Health 2017;5:e1221-e1234.

6. Solomon SD, Chew E, Duh EJ, Sobrin L, Sun JK, VanderBeek BL, et al. Diabetic retinopathy: a position statement by the American Diabetes Association. Diabetes Care 2017;40:412-418.

7. Ding J, Wong TY. Current epidemiology of diabetic retinopathy and diabetic macular edema. Curr Diab Rep 2012;12:346-354.

8. Ting DSW, Cheung GCM, Wong TY. Diabetic retinopathy: global prevalence, major risk factors, screening practices and public health challenges: a review. Clin Exp Ophthalmol 2016;44:260-277.

9. Caglar C, Demir E, Kucukler FK, Durmus M. A bibliometric analysis of academic publication on diabetic retinopathy disease trends during 1980-2014: a global and medical view. Int J Ophthalmol 2016;9:1663.

10. Publications UN, Programme UND. Human Development Report 2016: Human Development for Everyone: United Nations Publications; 2017.

11. Esteghamati A, Larijani B, Aghajani MH, Ghaemi F, Kermanchi J, Shahrami A, et al. Diabetes in Iran: prospective analysis from first nationwide diabetes report of National Program for Prevention and Control of Diabetes (NPPCD2016). Sci Rep 2017;7:13461.

12. Safaeipour M, Maveddat E. Assessment of areas with an emphasis on social indicators-economic and human development indicators in combination with the use of 
GIS techniques and TOPSIS. Studies on Urban Planning 2013;1:11-27 [In Persian].

13. Hatef E, Fotouhi A, Hashemi H, Mohammad K, Jalali KH. Prevalence of retinal diseases and their pattern in Tehran: the Tehran eye study. Retina 2008;28:755-762.

14. Amini M, Aminorroaya A, Safaei H, Behrooz Z, Teimori A. Prevalence of diabetic retinopathy in newly diagnosed type 2 diabetic patients in Isfahan, Iran. Acta Endocrinologica 2008;4:415-423.

15. Stroup DF, Berlin JA, Morton SC, Olkin I, Williamson $G D$, Rennie D, et al. Meta-analysis of observational studies in epidemiology: a proposal for reporting. JAMA 2000;283:2008-2012.

16. Salz DA, Witkin AJ. Imaging in diabetic retinopathy. Middle East Afr J Ophthalmol 2015;22:145.

17. Joanna Briggs Institute. Critical appraisal tools. Australia: The University of Adelaide; 2018 [cited 2018 Jun 01]. Available from: http://joannabriggs.org/research/criticalappraisal-tools.html

18. Haghdoost A, Hashemi H, Haji Aghajani M, Janbabaee G, Maher A, Noori Hekmat S, et al. Specialized and geographic distribution of specialists in Iran in 2016 and its estimates in 2026. Iran J Epidemiol 2018;13:122-132.

19. Brown LD, Cai TT, DasGupta A. Interval estimation for a binomial proportion. Stat Sci 2001;16:101-133.

20. Huedo-Medina TB, Sánchez-Meca J, Marín-Martínez F, Botella J. Assessing heterogeneity in meta-analysis: $Q$ statistic or I index? Psychol Methods 2006;11:193.

21. Janghorbani $M$, Amini $M$, Ghanbari $H$, Safaiee $H$. Incidence of and risk factors for diabetic retinopathy in Isfahan, Iran. Ophthalmic Epidemiol 2003;10:81-95.

22. Manaviat MR, Afkhami M, Shoja MR. Retinopathy and microalbuminuria in type II diabetic patients. BMC Ophthalmol 2004;4:9.

23. Abdollahi A, Daneshpazhooh $M$, Amirchaghmaghi $E$, Sheikhi S, Eshrati B, Bastanhagh M-H. Dermopathy and retinopathy in diabetes: is there an association? Dermatology 2007;214:133-136.

24. Abdollahi A, Malekmadani M, Mansoori M, Bostak A, Mirshahi MAA. Prevalence of diabetic retinopathy in patients with newly diagnosed type II diabetes mellitus. Acta Med Iran 2006;44:415-419.

25. Amini $M$, Safaei $H$, Aminorroaya $A$. The incidence of microalbuminuria and its associated risk factors in type 2 diabetic patients in Isfahan, Iran. Rev Diabet Stud 2007;4:242.

26. Manaviat M, Rashidi M, Afkhami-Ardekani M. Four years incidence of diabetic retinopathy and effective factors on its progression in type II diabetes. Eur J Ophthalmol 2008;18:572-577.

27. Gharaagaji R, Fagihzadeh S, Meshkani M, Rohipor R. The prevalence of diabetic retinopathy and related risk factors in diabetic type I, in Tehran, Iran. Res J Bio Sci 2008;3:596600.

28. Golbahar J, Rahimi M, Tabei MB, Aminzadeh MA. Clinical risk factors and association of hyperhomocysteinemia with diabetic retinopathy in Iranian type 2 diabetes patients: a cross-sectional study from Shiraz, Southern Iran. Diabetes Metab Syndr 2008;2:192-201.

29. Hosseini SM, Maracy MR, Amini M, Baradaran HR. A risk score development for diabetic retinopathy screening in Isfahan-Iran. J Res Med Sci 2009;14:105.
30. Javadi MA, Katibeh M, Rafati N, Dehghan MH, Zayeri $\mathrm{F}$, Yaseri $\mathrm{M}$, et al. Prevalence of diabetic retinopathy in Tehran province: a population-based study. BMC Ophthalmol 2009;9:12.

31. Soleymani A, Moazzezi Z, Gorjizadeh A. Frequency of ophthalmic complications on 140 cases of Type II diabetes mellitus, Babol-Iran. World App Sci J 2012;18:550-553.

32. Javanbakht M, Abolhasani F, Mashayekhi A, Baradaran HR. Health related quality of life in patients with type 2 diabetes mellitus in Iran: a national survey. PLOS ONE 2012;7:e44526.

33. Najafi L, Malek M, Valojerdi AE, Aghili R, Khamseh ME, Fallah $A E$, et al. Dry eye and its correlation to diabetes microvascular complications in people with type 2 diabetes mellitus. J Diabetes Complicat 2013;27:459-462.

34. Shaghaghi A, Ahmadi A, Matlabi H. Iranian patients require more pertinent care to prevent type 2 diabetes complications. Adv Prev Med 2014;2014:409391.

35. Gholamhossein $Y$, Behrouz H, Asghar Z. Diabetic retinopathy risk factors: plasma erythropoietin as a risk factor for proliferative diabetic retinopathy. Korean $\mathrm{J}$ Ophthalmol 2014;28:373-378.

36. Hosseini MS, Rostami Z, Saadat A, Saadatmand SM, Naeimi E. Anemia and microvascular complications in patients with type 2 diabetes mellitus. Nephrourol Mon 2014;6:e19976.

37. Maghbooli Z, Pasalar P, Keshtkar A, Farzadfar F, Larijani B. Predictive factors of diabetic complications: a possible link between family history of diabetes and diabetic retinopathy. J Diabetes Metab Disord 2014;13:55.

38. Tazhibi M, Sarrafzade S, Amini M. Retinopathy risk factors in type II diabetic patients using factor analysis and discriminant analysis. J Educ Health Promot 2014;3:85.

39. Ghodsi R, HamayeliMeharbani $H$, Avand A-Q, Bordbar A, Ahmadzadeh S. A study on the prevalence of diabetic complications in Fasa diabetes clinic. Asian J Med Pharm Res 2014;4:68-72.

40. Dehghan $\mathrm{MH}$, Katibeh $\mathrm{M}$, Ahmadieh $\mathrm{H}$, Nourinia R, Yaseri $M$. Prevalence and risk factors for diabetic retinopathy in the 40 to 80 year-old population in Yazd, Iran: The Yazd Eye Study. J Diabetes 2015;7:139-141.

41. Azizi-Soleiman F, Heidari-Beni M, Ambler G, Omar R, Amini M, Hosseini S-M. Iranian risk model as a predictive tool for retinopathy in patients with type 2 diabetes. Can $J$ Diabetes 2015;39:358-363.

42. Shamshirgaran SM, Ataei J, Alamdari MI, Safaeian A, Aminisani N. Predictors of health-related quality of life among people with type II diabetes Mellitus in Ardabil, Northwest of Iran, 2014. Prim Care Diabetes 2016;10:244250.

43. Rasoulinejad SA, Hajian-Tilaki K, Mehdipour E. Associated factors of diabetic retinopathy in patients that referred to teaching hospitals in Babol. Caspian J Intern Med 2015;6:224.

44. Mehravar F, Mansournia MA, Holakouie-Naieni K, NasliEsfahani E, Mansournia N, Almasi-Hashiani A. Associations between diabetes self-management and microvascular complications in patients with type 2 diabetes. Epidemiol Health 2016;38:e2016004.

45. Valizadeh R, Moosazadeh M, Bahaadini K, Vali L, Lashkari $\mathrm{T}$, Amiresmaili M. Determining the prevalence of retinopathy and its related factors among patients with type 
2 Diabetes in Kerman, Iran. Osong Public Health Res Perspect 2016;7:296-300.

46. Aidenloo NS, Mehdizadeh A, Valizadeh N, Abbaszadeh M, Qarequran S, Khalkhali H. Optimal glycemic and hemoglobin a1c thresholds for diagnosing diabetes based on prevalence of retinopathy in an iranian population. Iran Red Crescent Med J 2016;18:31254.

47. Hashemi H, Khabazkhoob M, Nabovati P, Ostadimoghaddam $H$, Shafaee S, Doostdar A, et al. The prevalence of age-related eye disease in an elderly population. Ophthalmic Epidemiol 2017;24:222-228.

48. Dehghan H, Charkazi A, Kouchaki GM, Zadeh BP, Dehghan BA, Matlabi M, et al. General self-efficacy and diabetes management self-efficacy of diabetic patients referred to diabetes clinic of Aq Qala, North of Iran. J Diabetes Metab Disord 2017;16:8.

49. Shamshirgaran S, Mamaghanian A, Aliasgarzadeh A, Aiminisani N, Iranparvar-Alamdari M, Ataie J. Age differences in diabetes-related complications and glycemic control. BMC Endocr Disord 2017;17:25.

50. Katibeh M, Behboudi H, Moradian S, Alizadeh Y, Beiranvand $\mathrm{R}$, Sabbaghi $\mathrm{H}$, et al. Rapid assessment of avoidable blindness and diabetic retinopathy in Gilan province, Iran. Ophthalmic epidemiol 2017;24:381-387.

51. Williams R, Airey M, Baxter H, Forrester J, KennedyMartin T, Girach A. Epidemiology of diabetic retinopathy and macular oedema: a systematic review. Eye (Lond) 2004;18:963.

52. Sivaprasad S, Gupta B, Crosby-Nwaobi R, Evans J. Prevalence of diabetic retinopathy in various ethnic groups: a worldwide perspective. Surv Ophthalmol 2012;57:347370.

53. French DD, Behrens JJ, Jackson KL, Kho AN, Walunas TL, Evans CT, et al. Payment reform needed to address health disparities of undiagnosed diabetic retinopathy in the city of Chicago. Ophthalmol Ther 2017;6:123-131.

54. Gibson DM. Eye care availability and access among individuals with diabetes, diabetic retinopathy, or age-related macular degeneration. JAMA Ophthalmol 2014;132:471477.

55. MacLennan PA, McGwin G, Heckemeyer C, Lolley VR, Hullett S, Saaddine J, et al. Eye care use among a highrisk diabetic population seen in a public hospital's clinics. JAMA Ophthalmol 2014;132:162-167.

56. Tajunisah I, Wong P, Tan L, Rokiah P, Reddy S. Awareness of eye complications and prevalence of retinopathy in the first visit to eye clinic among type 2 diabetic patients. Int J Ophthalmol 2011;4:519.

57. Gibson DM. The local availability of eye care providers and the vision health of adults in the United States. Ophthalmic Epidemiol 2016;23:223-231.

58. Thapa R, Twyana SN, Paudyal G, Khanal S, van Nispen $\mathrm{R}$, Tan S, et al. Prevalence and risk factors of diabetic retinopathy among an elderly population with diabetes in Nepal: the Bhaktapur Retina Study. Clin Ophthalmol 2018;12:561.

59. Pradeepa R, Anitha B, Mohan V, Ganesan A, Rema M. Risk factors for diabetic retinopathy in a South Indian type 2 diabetic population-the Chennai Urban Rural Epidemiology Study (CURES) Eye Study 4. Diabet Med 2008;25:536542.

60. Mumtaz SN, Fahim MF, Arslan M, Shaikh SA, Kazi U, Memon MS. Prevalence of diabetic retinopathy in Pakistan: a systematic review. Pak J Med Sci 2018;34:493.

61. Kanjee R, Dookeran RI, Mathen MK, Stockl FA, Leicht R. Six-year prevalence and incidence of diabetic retinopathy and cost-effectiveness of tele-ophthalmology in Manitoba. Can J Ophthalmol 2016;51:467-470.

62. Vila L, Viguera J, Aleman R. Diabetic retinopathy and blindness in Spain: epidemiology and prevention. Endocrinol Nutr 2008;55:459-475.

63. Bourne RR, Stevens GA, White RA, Smith JL, Flaxman SR, Price $\mathrm{H}$, et al. Causes of vision loss worldwide, 1990-2010: a systematic analysis. Lancet Glob Health 2013;1:e339e349.

64. Zheng $\mathrm{Y}, \mathrm{He} \mathrm{M}$, Congdon $\mathrm{N}$. The worldwide epidemic of diabetic retinopathy. Indian J Ophthalmol 2012;60:428.

65. Romero-Aroca P, Navarro-Gil R, Valls-Mateu A, SagarraAlamo R, Moreno-Ribas A, Soler N. Differences in incidence of diabetic retinopathy between type 1 and 2 diabetes mellitus: a nine-year follow-up study. $\mathrm{Br} J \mathrm{Oph}-$ thalmol 2017;101:1346-1351.

66. Romero-Aroca P, Fernàndez-Alart J, Baget-Bernaldiz M, Méndez-Marín I, Salvat-Serra M. Diabetic retinopathy epidemiology in type II diabetic patients. Effect of the changes in the diagnostic criteria and stricter control of the diabetes between 1993 and 2005 on the incidence of diabetic retinopathy. Arch Soc Esp Oftalmol 2007;82:209-218.

67. Raman R, Ganesan S, Pal SS, Gella L, Kulothungan V, Sharma T. Incidence and progression of diabetic retinopathy in urban India: Sankara Nethralaya-Diabetic Retinopathy Epidemiology and Molecular Genetics Study (SNDREAMS II), Report 1. Ophthalmic Epidemiol 2017;24:294302.

68. Song SJ, Han K, Choi KS, Ko SH, Rhee Ej, Park CY, et al. Trends in diabetic retinopathy and related medical practices among type 2 diabetes patients: results from the National Insurance Service Survey 2006-2013. J Diabetes Investig 2018;9:173-178.

69. Bek $T$, Lund-Andersen $H$, Hansen $A B$, Johnsen $K B$, Sandbæk A, Lauritzen T. The prevalence of diabetic retinopathy in patients with screen-detected type 2 diabetes in Denmark: the ADDITION study. Acta Ophthalmol 2009;87:270-274.

70. Ruta L, Magliano D, Lemesurier R, Taylor H, Zimmet P, Shaw J. Prevalence of diabetic retinopathy in type 2 diabetes in developing and developed countries. Diabet Med 2013;30:387-398.

71. Sikorski BL, Malukiewicz G, Stafiej J, Lesiewska-Junk H, Raczynska D. The diagnostic function of OCT in diabetic maculopathy. Mediators Inflamm 2013;2013;434560. 